\title{
Approximate bandstructures of semiconductor alloys from tight-binding supercell calculations
}

\author{
Timothy B Boykin ${ }^{1}$, Neerav Kharche ${ }^{2}$, Gerhard Klimeck ${ }^{2}$ and \\ Marek Korkusinski ${ }^{2}$ \\ ${ }^{1}$ Department of Electrical and Computer Engineering, The University of Alabama in Huntsville, \\ Huntsville, AL 35899, USA \\ ${ }^{2}$ Network for Computational Nanotechnology, School of Electrical and Computer Engineering, \\ Purdue University, West Lafayette, IN 47907, USA
}

Received 16 October 2006

Published 5 January 2007

Online at stacks.iop.org/JPhysCM/19/036203

\begin{abstract}
Alloys such as AlGaAs, InGaAs, and SiGe find widespread usage in nanoelectronic devices such as quantum dots and nanowires. For these devices, in which the carriers probe nanometre-scale local disorder, the commonly employed virtual crystal approximation (VCA) is inadequate. Although the VCA produces small-cell $E(\mathbf{k})$ relations it fails to include local disorder. In contrast, random-alloy supercell calculations do include local disorder but only deliver band extrema and supercell (not small cell) $E(\mathbf{k})$ relations. Smallcell $E(\mathbf{k})$ relations are, however, needed to interpret transport parameters such as effective masses. This work presents a method to extract the necessary approximate small-cell $E(\mathbf{k})$ relations from the disordered supercell states. The method is applied to AlGaAs and gives significantly improved energy gaps versus the VCA, as well as approximate effective masses. The results illuminate the bowing of the $\Gamma$-valley gap and the good agreement with bulk experimental data shows that this method is well suited for nanodevices.
\end{abstract}

\section{Introduction}

The empirical tight-binding method is ideally suited for modelling systems with atomically sharp interfaces or local disorder for which plane-wave methods are inefficient. If some part of the geometry is of a repeated nature such as segments of a quantum wire or a quantum well, or an array of quantum dots, a significant device portion can be represented in a large supercell which can contain tens of millions of atoms [1]. That supercell may then be repeated in one, two, or three dimensions. Example calculations include quantum dots [1], impurities [2], nanowires [3], nano-FETs [4], and alloys [5]. Tight-binding electronic structure calculations have demonstrated the capability to handle tens of millions of atoms [1] due to their reduced computational requirements compared to pseudopotential methods [6] which have been limited to one or two million atoms. Plane-wave methods require extremely large numbers of waves 
to resolve sharp interfaces. In contrast, the structure geometry is irrelevant in tight binding because the number of basis states is solely determined by the number of atoms and the atomic orbital basis.

Frequently, technically relevant systems are imperfect. Examples include quantum dots or wires made of alloys or having rough surfaces, nano-FETs with inhomogeneous strain distributions, or simply three-dimensional random alloys. For such disordered systems the small cells differ and significant device portions must be treated in a much larger supercell. The use of large supercells creates both a technical problem and a conceptual problem. The technical problem arises even for identical small cells: how does one extract a smallcell bandstructure from the multiply-folded supercell bandstructure? This technical problem has been solved in [7]. The conceptual problem remains: does an experimentally relevant bandstructure exist when the small cells are not all identical, and if so, how does one extract it from a large supercell calculation? This question goes beyond that of the minimum random AlGaAs supercell necessary to extract meaningful conduction- and valence-band edges, addressed in [5]. In view of the fact that experiments have measured bandgaps and effective masses of alloys, one would expect that relevant small-cell $E(\mathbf{k})$ relations should be obtainable from supercell calculations. The theory behind approximate small-cell $E(\mathbf{k})$ relations and the process by which they may be obtained are the subjects of this paper.

The importance of small-cell $E(\mathbf{k})$ relations for random alloys is best seen in the context of traditional supercell calculations. Supercell calculations previously could only compute the lowest conduction-band energy and the highest valence-band energy. They could not deliver any other transport parameters (effective masses, other extrema), nor could they address the reasons for the failure of the VCA to properly represent the alloy bandgap. The method presented here addresses both of these shortcomings. Furthermore, it is also applicable to lower-dimensional structures such as quantum wells and nanowires. The method is especially relevant for nanodevices since one can estimate device performance via a 'top of barrier model' [8] from band edges, dispersion and effective masses that greatly impact device design under non-idealities such as disorder from alloys or interfaces.

Traditional approaches to the problem of approximate $E(\mathbf{k})$ relations in disordered systems involve defining an approximate alloy Hamiltonian. Examples include the VCA and the coherent-potential approximation (CPA) [9]. While the VCA fails to incorporate true randomness, more recent first-principles methods such as the locally self-consistent multiple scattering method [10] and the KKR-nonlocal CPA dynamical cluster method [11] do capture nanometre-scale random variations. The difficulty with these new approaches is that they are often far too costly for use with realistically extended nanostructures. For such large systems the empirical tight-binding approach is generally the full-bandstructure method of choice, due to its good balance between accuracy and computational efficiency. A method for calculating approximate small-cell $E(\mathbf{k})$ relations, uniquely tailored to the tight-binding supercell technique, is therefore highly desirable.

Here we present such a method, a major extension and adaptation of our zoneunfolding technique for perfect supercells [7]. Unlike traditional approximate bandstructure approaches, which define a translationally symmetric approximate Hamiltonian a priori, our new work attacks the problem in reverse. That is, our method projects out of the disordered supercell states the best translationally symmetric approximate Hamiltonian. While projection techniques have been used in supercell calculations, our method differs from earlier efforts such as those of Wang et al [6] and Dagram et al [12] in two important respects. First, these earlier efforts concentrate on the spectral composition of a specific supercell state (often a band extremum or quantum well ground state). We consider the opposite problem: what are the average energies for a given small-cell wavevector? Second, earlier approaches do not 
attempt to extract small-cell bandstructures from supercell calculations. We do, by examining the cumulative probability at a fixed small-cell wavevector, which should be step-like when an approximate small-cell bandstructure description is appropriate. This cumulative probability is analogous to the integral over energy of the Bloch spectral density function of true randomalloy electronic structure calculations [9].

We apply this method to the AlGaAs bulk alloy problem, since the case of threedimensional small-cell wavevectors is the most general. The application of the method to cases of one- or two-dimensional wavevectors is straightforward. We demonstrate that the approximate bands and gaps projected from random-alloy supercells fit experiment much better than do those calculated with the VCA. The method can thus provide reassurance that the tightbinding supercell alloy calculation reproduces good three-dimensional alloy behaviour.

The paper is organized as follows: section 2 briefly reviews the projection method, then further develops it for the case of imperfect supercells; section 3 presents our results on AlGaAs random-alloy supercells; and section 4 provides our conclusions.

\section{Method}

\subsection{General}

One computes approximate bandstructures for random alloys by analysing random-alloy supercell eigenvectors. In the supercell calculation, the Bloch condition is enforced only over the supercell, not over its constituent primitive cells (for a random alloy these primitive cells are not all identical). The supercell Hamiltonian has one basis state for each atom and orbital in the supercell, so that disorder is treated on an atomistic level. Specifically, nearest-neighbour interaction parameters are assigned individually for each bond in the supercell. Thus, random atomic distributions and displacements are fully included, but restricted to within the chosen supercell. Supercell eigenvalues and eigenvectors are used in the band-projection algorithm.

The band projection method for imperfect supercells is an extension of the zone-unfolding procedure of [7] for perfect supercells. The solid considered has $N_{\mathrm{S}}$ supercells, located at positions $\mathbf{R}_{j}$, and each supercell contains $N_{\mathrm{C}}$ small (i.e., primitive) cells, located at positions $\boldsymbol{\rho}_{l}$ relative to the respective supercell origin. The wavefunction is described in terms of a localized orbital basis with $N_{\mathrm{O} \text {,cell }}$ orbitals per small cell, which are indexed by orbital type $\alpha$ (s, $\mathrm{p}_{x}$, etc) and atom within the small cell $\mu$ (for crystals with polyatomic bases). The supercell eigenstate of energy $E_{p}$ is written as

$$
\left|\Psi_{p}(\mathbf{K})\right\rangle=\sum_{j=1}^{N_{\mathrm{S}}} \sum_{\alpha, \mu}^{N_{\mathrm{O}, \text { cell }}} \sum_{l=1}^{N_{\mathrm{C}}} \frac{\mathrm{e}^{\mathrm{i} \mathbf{K} \cdot \mathbf{R}_{j}}}{\sqrt{N_{\mathrm{S}}}} \beta_{l, p}^{(\alpha, \mu)}(\mathbf{K})\left|\alpha, \mu ; \mathbf{R}_{j}+\boldsymbol{\rho}_{l}\right\rangle
$$

where $\mathbf{K}$ is a wavevector belonging to the supercell first Brillouin zone.

Because the whole concept of an approximate bandstructure becomes questionable if the disorder is too great we make two assumptions in writing equation (1). First, we assume that the concept of identically sized small cells is meaningful. That is, we treat cases where it is possible to divide the supercell into small cells of identical size, but which may differ in other respects such as composition (as in the $\mathrm{AlGaAs}$ random alloys treated below) or the positions of the atoms within the small cell (as in a strained alloy like SiGe). Second, we employ the same set of atomic-like orbitals $\left\{\left|\alpha, \mu ; \mathbf{R}_{j}+\boldsymbol{\rho}_{l}\right\rangle\right\}$ in each small cell. Thus the randomness within the supercell appears in the matrix elements of the Hamiltonian via the potential function (as opposed to the basis set) and is expressed in equation (1) in the coefficients $\beta_{l, p}^{(\alpha, \mu)}(\mathbf{K})$, which for different small cells now generally differ in both magnitude and phase. We remark that in the empirical tight-binding method one has only Hamiltonian matrix elements and not explicit 
orbital functions. Hence, lengths smaller than the interatomic spacing cannot be resolved and the use of the same atomic-like functions in each small cell causes no difficulty. These assumptions are not particularly restrictive, since if either one fails the whole concept of an approximate bandstructure becomes problematic.

To find the translationally symmetric Hamiltonian which mimics the true, imperfect system as closely as possible via the projection method, we define a Bloch-state basis of small-cell wavevector $\mathbf{k}=\mathbf{K}+\mathbf{G}_{\mathbf{n}}$, where $\mathbf{G}_{\mathbf{n}}$ is a supercell reciprocal lattice vector. A small-cell Bloch state of energy $E_{\eta}$ with wavevector $\mathbf{k}=\mathbf{K}+\mathbf{G}_{\mathbf{n}}$ may therefore be written:

$$
\left|\psi_{\eta}\left(\mathbf{K}+\mathbf{G}_{\mathbf{n}}\right)\right\rangle=\sum_{j=1}^{N_{\mathrm{S}}} \sum_{\alpha, \mu}^{N_{\mathrm{O}, \text { cell }}} \sum_{l=1}^{N_{\mathrm{C}}} \frac{\mathrm{e}^{\mathrm{i} \mathbf{K} \cdot \mathbf{R}_{j}}}{\sqrt{N_{\mathrm{S}} N_{\mathrm{C}}}} b_{\eta}^{(\alpha, \mu)}\left(\mathbf{K}+\mathbf{G}_{\mathbf{n}}\right) \mathrm{e}^{\mathrm{i}\left(\mathbf{K}+\mathbf{G}_{\mathbf{n}}\right) \cdot \boldsymbol{\rho}_{l}}\left|\alpha, \mu ; \mathbf{R}_{j}+\boldsymbol{\rho}_{l}\right\rangle .
$$

We remark that at each small-cell wavevector there are $N_{\mathrm{O} \text {,cell }}$ small-cell bands (i.e., the same as the number of atomic-like orbitals per cell). Note that the exact determination of the $\mathbf{G}_{\mathbf{n}}$ depends upon the supercell geometry. If the supercell contains a fixed number of small cells in each of the (generally non-orthogonal) directions corresponding to small-cell primitive direct lattice vectors, then the method of [7] gives the $\mathbf{G}_{\mathbf{n}}$. If, on the other hand, the supercell is not commensurate with a fixed number of small cells along each small-cell primitive direct lattice vector, a different procedure, such as that given in [13], is necessary.

Because the supercell states, equation (1) and the small-cell states, equation (2) are linear combinations of the same set of atomic-like orbitals, these states must be related by a basis transformation. In an imperfect system the supercell state is generally a superposition of all small-cell Bloch states:

$$
\left|\Psi_{p}(\mathbf{K})\right\rangle=\sum_{\eta=1}^{N_{\mathrm{O}, \mathrm{cell}}} \sum_{j=1}^{N_{\mathrm{C}}} a_{p ; \eta, \mathbf{n}(j)}\left|\psi_{\eta}\left(\mathbf{K}+\mathbf{G}_{\mathbf{n}(j)}\right)\right\rangle .
$$

As in [7], one may project the small-cell states out of the supercell state. Taking the inner product of equation (3) with the bra $\left\langle\alpha, \mu ; \mathbf{R}_{j}+\rho_{l}\right|$ and rearranging yields one of the $N_{\mathrm{C}}$ equations:

$$
\mathrm{e}^{-\mathrm{i} \mathbf{K} \cdot \rho_{l}} \beta_{l, p}^{(\alpha, \mu)}(\mathbf{K})=\frac{1}{\sqrt{N_{\mathrm{C}}}} \sum_{j=1}^{N_{\mathrm{C}}} \sum_{\eta=1}^{N_{\mathrm{O}, \text { cell }}} a_{p ; \eta, \mathbf{n}(j)} b_{\eta}^{(\alpha, \mu)}\left(\mathbf{K}+\mathbf{G}_{\mathbf{n}(j)}\right) \mathrm{e}^{\mathrm{i} \mathbf{G}_{\mathbf{n}(j)} \cdot \boldsymbol{\rho}_{l}} .
$$

In matrix form, these equations (one per primitive cell) read

$$
\mathbf{B}_{p}^{(\alpha, \mu)}(\mathbf{K})=\underline{\mathbf{U}} \cdot \mathbf{C}_{p}^{(\alpha, \mu)}(\mathbf{K})
$$

where

$$
\begin{aligned}
& \mathbf{B}_{p}^{(\alpha, \mu)}(\mathbf{K})=\left[\begin{array}{c}
\mathrm{e}^{-\mathrm{i} \mathbf{K} \cdot \rho_{1}} \beta_{1, p}^{(\alpha, \mu)}(\mathbf{K}) \\
\vdots \\
\mathrm{e}^{-\mathrm{i} \mathbf{K} \cdot \rho_{N_{\mathrm{C}}}} \beta_{N_{\mathrm{C}}, p}^{(\alpha, \mu)}(\mathbf{K})
\end{array}\right], \\
& \mathbf{C}_{p}^{(\alpha, \mu)}(\mathbf{K})=\left[\begin{array}{c}
\sum_{\eta=1}^{N_{\mathrm{O}, \text { cell }}} a_{p ; \eta, \mathbf{n}(1)} b_{\eta}^{(\alpha, \mu)}\left(\mathbf{K}+\mathbf{G}_{\mathbf{n}(1)}\right) \\
\vdots \\
\sum_{\eta=1}^{N_{\text {O,cell }}} a_{p ; \eta, \mathbf{n}\left(N_{\mathrm{C}}\right)} b_{\eta}^{(\alpha, \mu)}\left(\mathbf{K}+\mathbf{G}_{\mathbf{n}\left(N_{\mathrm{C}}\right)}\right)
\end{array}\right]
\end{aligned}
$$

and the $N_{\mathrm{C}} \times N_{\mathrm{C}}$ unitary [7] matrix $\underline{\mathbf{U}}$ is

$$
\underline{\mathbf{U}}=\frac{1}{\sqrt{N_{\mathrm{C}}}}\left[\begin{array}{cccc}
\mathrm{e}^{\mathrm{i} \rho_{1} \cdot \mathbf{G}_{\mathbf{n}(1)}} & \mathrm{e}^{\mathrm{i} \rho_{1} \cdot \mathbf{G}_{\mathbf{n}(2)}} & \cdots & \mathrm{e}^{\mathrm{i} \rho_{1} \cdot \mathbf{G}_{\mathbf{n}\left(N_{\mathrm{C}}\right)}} \\
\mathrm{e}^{\mathrm{i} \rho_{2} \cdot \mathbf{G}_{\mathbf{n}(1)}} & \ddots & \ddots & \mathrm{e}^{\mathrm{i} \rho_{2} \cdot \mathbf{G}_{\mathbf{n}\left(N_{\mathrm{C}}\right)}} \\
\vdots & \ddots & \ddots & \vdots \\
\mathrm{e}^{\mathrm{i} \rho_{N_{\mathrm{C}}} \cdot \mathbf{G}_{\mathbf{n}(1)}} & \cdots & \cdots & \mathrm{e}^{\mathrm{i} \rho_{N_{\mathrm{C}}} \cdot \mathbf{G}_{\mathbf{n}\left(N_{\mathrm{C}}\right)}}
\end{array}\right]
$$




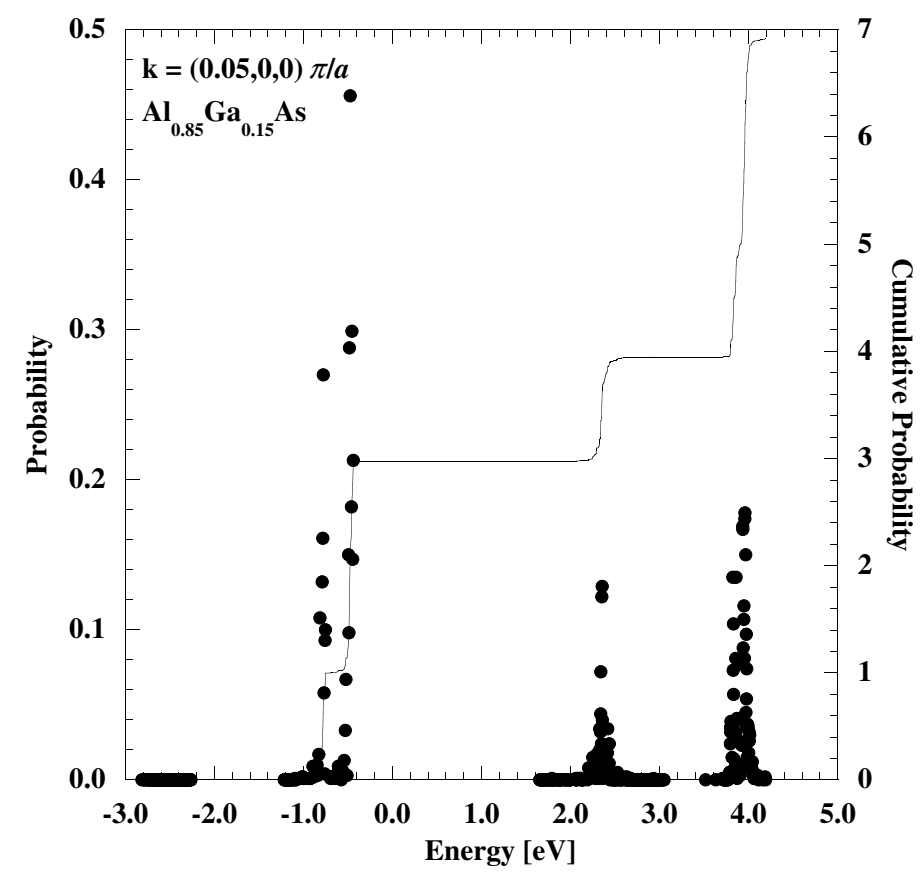

Figure 1. Probability (symbols) and cumulative probability (solid line) versus supercell-eigenstate energy, $E_{p}$, for the $40 \times 2 \times 2 \mathrm{Al}_{0.85} \mathrm{Ga}_{0.15}$ As random-alloy supercell at $\mathbf{K}=\mathbf{0}, \mathbf{k}=\mathbf{G}=$ $(0.05,0,0) \pi / a$. Here the algorithm could resolve a state at about $2.46 \mathrm{eV}$.

As in [7], equation (5) is repeatedly solved for each (atom, orbital) pair $(\alpha, \mu)$ and the results are saved. Taking advantage of the normalization of the Bloch states yields the following relation:

$$
\begin{aligned}
\mathscr{P}_{p ; \mathbf{n}(j)} & =\sum_{\eta=1}^{N_{\text {Oocell }}}\left|a_{p ; \eta, \mathbf{n}(j)}\right|^{2}=\sum_{\alpha, \mu}^{N_{\text {o,cell }}}\left|\left[\mathbf{C}_{p}^{(\alpha, \mu)}\right]_{j}\right|^{2}, \\
{\left[\mathbf{C}_{p}^{(\alpha, \mu)}\right]_{j} } & =\sum_{\eta=1}^{N_{\text {O,cell }}} a_{p ; \eta, \mathbf{n}(j)} b_{\eta}^{(\alpha, \mu)}\left(\mathbf{K}+\mathbf{G}_{\mathbf{n}(j)}\right) .
\end{aligned}
$$

In the case of an imperfect supercell, the existence of an approximate small-cell bandstructure suggests that in the case of nondegenerate bands, at each $\mathbf{k}=\mathbf{K}+\mathbf{G}_{\mathbf{n}}$ the first sum in equation (8) will be dominated by one of the $\left|a_{p ; \eta, \mathbf{n}(j)}\right|^{2}$.

The $\mathscr{P}_{p ; \mathbf{n}(j)}$ are computed and saved for all supercell states, $p$, and all wavevectors $\mathbf{k}=\mathbf{K}+\mathbf{G}_{\mathbf{n}}$. These sums represent the probability of there being a small-cell Bloch state at energy $E_{p}$ and wavevector $\mathbf{k}=\mathbf{K}+\mathbf{G}_{\mathbf{n}}$. The spectrum (for fixed wavevector) is used in band determination, discussed more fully in the following subsection. One example of such a spectrum is shown in figure 1, which also shows a plot of the cumulative probability. At fixed wavevector the probability tends to peak at energies corresponding to approximate small-cell bands. We note that this approach to the probability function is closely related to the Bloch spectral density function of true random-alloy electronic structure calculations [9]. Here definite peaks are evident, with corresponding steps in the cumulative probability. These features suggest that a band may be defined at each step and the next subsection develops the detailed physics behind this insight. We note that besides having the best physical justification, step detection is algorithmically much simpler than is peak finding. 


\subsection{Probability sum rule and band-counting}

The probability sum rule relates $\mathscr{P}_{p ; \mathbf{n}(j)}$ (i.e., the sum of all the $\left|a_{p ; \eta, \mathbf{n}(j)}\right|^{2}$ at fixed small-cell wavevector) to the number of bands in the Bloch basis. It is derived beginning with equation (3), from which one finds

$$
\left|a_{p ; \eta, \mathbf{n}(j)}\right|^{2}=\left\langle\psi_{\eta}\left(\mathbf{K}+\mathbf{G}_{\mathbf{n}(j)}\right) \mid \Psi_{p}(\mathbf{K})\right\rangle\left\langle\Psi_{p}(\mathbf{K}) \mid \psi_{\eta}\left(\mathbf{K}+\mathbf{G}_{\mathbf{n}(j)}\right)\right\rangle .
$$

Summing over all $N_{\mathrm{O} \text {,cell }}$ values of $\eta$ and all $N_{\mathrm{C}} N_{\mathrm{O} \text {, cell }}$ values of $p$, and using the closure relation for a fixed supercell wavevector, $\mathbf{K}$ (recall that the supercell and small-cell states are linear combinations of the same atomic-like orbitals),

$$
1_{\mathbf{K}}=\sum_{p=1}^{N_{\mathrm{C}} N_{\mathrm{O}, \mathrm{cell}}}\left|\Psi_{p}(\mathbf{K})\right\rangle\left\langle\Psi_{p}(\mathbf{K})\right|
$$

one finds

$$
\begin{aligned}
\sum_{p=1}^{N_{\mathrm{C}} N_{\mathrm{O}, \text { cell }}} \sum_{\alpha, \mu}^{N_{\mathrm{O}, \text { cell }}}\left|\left[\mathbf{C}_{p}^{(\alpha, \mu)}\right]_{j}\right|^{2}=\sum_{p=1}^{N_{\mathrm{C}} N_{\mathrm{O}, \text { cell }}} \sum_{\eta=1}^{N_{\mathrm{O}, \text { cell }}}\left|a_{p ; \eta, \mathbf{n}(j)}\right|^{2} \\
=\sum_{\eta=1}^{N_{\text {O,cell }}}\left\langle\psi_{\eta}\left(\mathbf{K}+\mathbf{G}_{\mathbf{n}(j)}\right) \mid \psi_{\eta}\left(\mathbf{K}+\mathbf{G}_{\mathbf{n}(j)}\right)\right\rangle=N_{\mathrm{O}, \text { cell }}
\end{aligned}
$$

where we employ the normalization of the small-cell Bloch states in the last step. Equation (11) thus establishes that at each wavevector $\mathbf{k}=\mathbf{K}+\mathbf{G}_{\mathbf{n}}$ there are $N_{\mathrm{O} \text {,cell }}$ bands. It is therefore reasonable to take as the definition of a band a unit step in the cumulative probability; larger integral steps of height $n$ indicate $n$-fold degenerate or near-degenerate bands. Of course the steps will not be perfectly sharp for imperfect systems, but if they are still fairly sharp the association of a step in the cumulative probability with a small-cell band is physically wellfounded. Note that searching for steps eliminates problems which can occur when two or more probability peaks whose total is unity are closely spaced.

Our algorithm for determining the approximate small-cell bands from an imperfect supercell is summarized below; additional comments on the calculation may be found in the appendix.

(i) Choose a small-cell wavevector $\mathbf{k}=\mathbf{K}+\mathbf{G}_{\mathbf{n}}$ at which to perform projections.

(ii) Compute and save the probability sums, $\mathscr{P}_{p ; \mathbf{n}}$, generated by repeated projections, equation (5), out of each supercell state of energy $E_{p}$. A convenient method is to make the $\mathscr{P}_{p ; \mathbf{n}}$ elements of a matrix indexed by supercell energy $E_{p}$ and small-cell wavevector $\mathbf{k}=\mathbf{K}+\mathbf{G}_{\mathbf{n}}$.

(iii) Search for steps in the cumulative probability as a function of the energy, $E_{p}$.

(iv) Using the energies $E_{p}$ and corresponding probability sums, $\mathscr{P}_{p ; \mathbf{n}}$, compute the mean energies $\bar{E}$ and standard deviations $\Delta E$ between successive steps in the cumulative probability.

Our algorithm has three control parameters: the minimum resolvable gap; the minimum recognizable probability sum; and the minimum band probability. Probability sums for energies separated by less than the minimum resolvable gap are treated as belonging to the same smallcell band. Probability sums less than the minimum recognizable value are discarded. A band is counted only after the cumulative probability has increased by the minimum band probability. These control parameters are especially helpful when not all of the supercell eigenstates are available, as often happens when the supercell Hamiltonian is diagonalized by a method such as Lanczos [14]. 
In figure 1 the control parameters are a minimum gap of $0.50 \mathrm{eV}$, a minimum probability of 0.001 , and a minimum band probability of 0.50 . Note the step in the cumulative probability of very nearly 1.0 at around $2.46 \mathrm{eV}$. Here the algorithm resolved the lowest conduction band. In the valence bands, the split-off holes (at around $-0.8 \mathrm{eV}$ ) are clearly differentiated from the heavy and light holes. Even though $\mathbf{k}=0.05(\pi / a) \mathbf{e}_{x}$, the heavy and light holes (at around $-0.6 \mathrm{eV}$ ) are not well differentiated. Note the sharp step in cumulative probability from about 1 to 3 . The algorithm could not separate the two bands and reported an effectively doubly degenerate band at this energy and wavevector. Finally we note that this approach differs from earlier projection calculations $[6,12]$. These earlier efforts concentrate on the smallcell wavevector contribution to a fixed supercell state. Here we do the opposite, plotting the supercell state contributions to states of fixed small-cell wavevector.

\section{Results}

We employ the method of section 2 to calculate the approximate bands for $\mathrm{Al}_{x} \mathrm{Ga}_{1-x} \mathrm{As}$ random alloys, which we compare to the bands calculated with the VCA. The supercell calculations are performed with NEMO-3D [1] $]^{3}$. As discussed in section 2, the supercell calculation assigns tight-binding parameters for each bond in the supercell separately. In the present calculation, this means that AlAs nearest-neighbour parameters are used for each As-Al bond and GaAs nearest-neighbour parameters are used for each As-Ga bond. The onsite parameters for each As atom are determined separately as a weighted average of the As onsite parameters for GaAs and AlAs, depending upon the nearest-neighbour atom (Ga or Al). Thus each As atom in the supercell can have one of five different onsite energies between the extremal values in the GaAs and AlAs lists. The random environment is therefore fully included in both the onsite and nearest-neighbour interactions of the supercell Hamiltonian. Since the AlGaAs lattice constant is virtually identical to the constituent GaAs and AlAs materials, strain is negligible and there is no disorder in atom positions.

The fundamental building block for zinc blende in NEMO-3D is the face-centred cubic (FCC) conventional unit cell, a cube of side $a$ containing four small cells (rhombohedra), or 8 atoms. Since this results in a shape which is not commensurate with a fixed number of small cells along each of the primitive direct lattice vectors, the method of [13] is used to find the allowed small-cell wavevectors, $\mathbf{k}=\mathbf{K}+\mathbf{G}$. For a supercell geometry such as that studied here with $N_{\alpha}, \alpha \in\{x, y, z\}$ cubes along each of the Cartesian axes, the supercell reciprocal lattice vectors are given by

$$
\begin{aligned}
& \mathbf{G}=\frac{2 \pi}{a}\left[\frac{m_{x}}{N_{x}} \mathbf{e}_{x}+\frac{m_{y}}{N_{y}} \mathbf{e}_{y}+\frac{m_{z}}{N_{z}} \mathbf{e}_{z}\right]+\mathbf{q}_{j} \\
& m_{\alpha}=\left\{\begin{array}{l}
-\frac{\left(N_{\alpha}-1\right)}{2}, \ldots, \frac{\left(N_{\alpha}-1\right)}{2} ; \quad N_{\alpha} \text { odd } \\
-\frac{\left(N_{\alpha}-2\right)}{2}, \ldots, \frac{N_{\alpha}}{2} ; \quad N_{\alpha} \text { even } \\
\mathbf{q}_{0}=\mathbf{0}, \quad \mathbf{q}_{1}=\frac{2 \pi}{a} \mathbf{e}_{x}, \quad \mathbf{q}_{2}=\frac{2 \pi}{a} \mathbf{e}_{y}, \quad \mathbf{q}_{3}=\frac{2 \pi}{a} \mathbf{e}_{z} .
\end{array}\right.
\end{aligned}
$$

The three nonzero $\mathbf{q}_{j}$ all lie within the small-cell (FCC) first Brillouin zone and are necessary, since each cube contains four FCC primitive cells, and therefore contributes four independent small-cell wavevectors. Note that it may be necessary to shift the small-cell wavevectors

\footnotetext{
3 NEMO 3-D development is being continued by the Network for Computational Nanotechnology (NCN). Simulations were performed on nanoHUB.org resources. nanoHUB.org is a web site dedicated to advancing nanotechnology through theory, modelling, and simulation for research and education.
} 


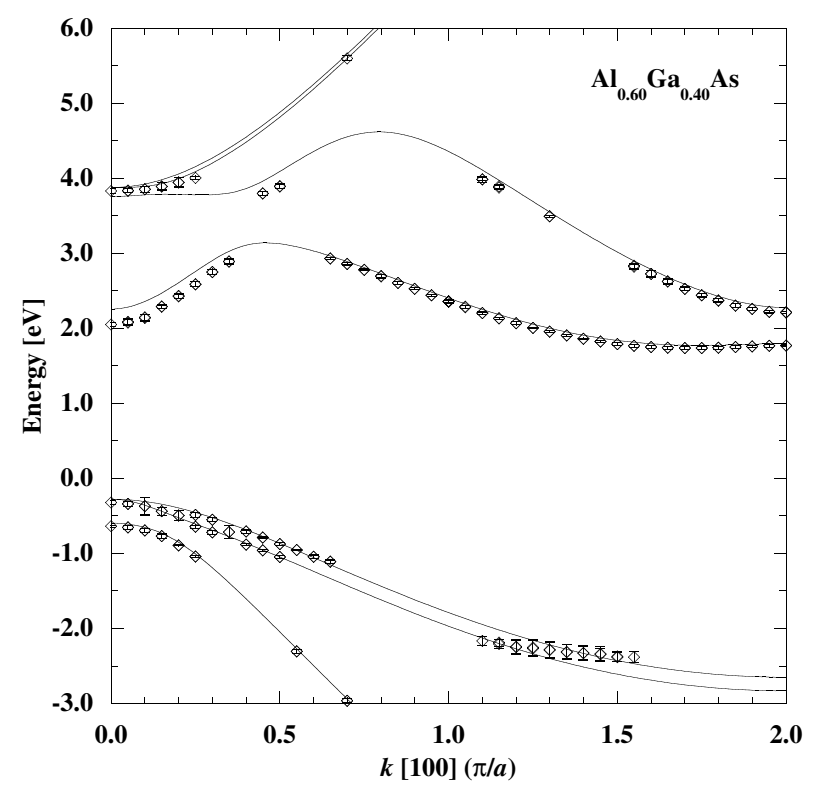

Figure 2. Approximate bands with error bars projected out of the $40 \times 2 \times 2 \mathrm{Al}_{0.60} \mathrm{Ga}_{0.40} \mathrm{As}$ random-alloy supercell (diamonds) and the VCA bands (solid lines) for bulk $\mathrm{Al}_{0.60} \mathrm{Ga}_{0.40} \mathrm{As}$. Note the significantly lower conduction-band minimum at $\mathbf{k}=\mathbf{0}$ in the random-alloy case.

$\mathbf{k}=\mathbf{K}+\mathbf{G}$ back into the small-cell (here FCC) first Brillouin zone. All supercell calculations are performed for supercell wavevector $\mathbf{K}=\mathbf{0}$ and the supercell is rectangular, $N_{x} \times N_{y} \times N_{z}=$ $40 \times 2 \times 2$ cubes $(640$ small cells $=1280$ atoms $)$. This shape gives many small-cell wavevectors along [100], the direction with the most interesting and relevant bandstructure features for $\mathrm{Al}_{x} \mathrm{Ga}_{1-x}$ As.

In contrast, the VCA treats the alloy as a pseudo-material, in which all anions are the same (here, As), as are all cations (here $\mathrm{Al}_{x} \mathrm{Ga}_{1-x}$ pseudo-atoms). The $\mathrm{Al}_{x} \mathrm{Ga}_{1-x} \mathrm{As} \mathrm{VCA}$ parameters, which are inputs to the standard bulk (i.e., two-atom) Hamiltonian, are calculated in the usual manner,

$$
P_{\mathrm{AlGaAs}}(x)=x P_{\mathrm{AlAs}}+(1-x) P_{\mathrm{GaAs}}
$$

where $P$ is a tight-binding parameter (either onsite or nearest-neighbour). Thus, the VCA does not incorporate randomness at an atomistic level, unlike the supercell calculation which is used in our approximate bandstructure calculation.

The underlying tight-binding model for all calculations is the $\mathrm{sp}^{3} \mathrm{~d}^{5} \mathrm{~s}^{*}$ model [15], and the GaAs and AlAs parameters used for both calculations are listed in table 1 in the SlaterKoster [16] notation. Selected gaps and effective masses reproduced by these parameters are listed in table 2, along with experimental values [17], where available. The parameters were optimized using our genetic algorithm [18]. We remark that another exhaustive source for AlGaAs data is Adachi's review paper [19]. The values for effective masses and bandgaps reported there are in good agreement with those reported in [17]. In addition to these sources, we used the experimental results of Lee et al [20] on the variation of the $\Gamma$-valley bandgap and mass with composition.

Figure 2 (for $\mathrm{Al}_{0.6} \mathrm{Ga}_{0.4} \mathrm{As}$ ) shows an example of the approximate small-cell bands projected out of the random-alloy supercell eigenstates (open diamonds with error bars) 
Table 1. Diagonal and spin-orbit parameters, and two-centre integrals for our parameterizations of GaAs and AlAs in the $\mathrm{sp}^{3} \mathrm{~d}^{5} \mathrm{~s}^{*}$ model (in $\mathrm{eV}$ ).

\begin{tabular}{|c|c|c|}
\hline Parameter & GaAs & AlAs \\
\hline$E_{\mathrm{s} a}$ & -5.500420 & -5.17012 \\
\hline$E_{\mathrm{p} a}$ & 4.151070 & 4.39708 \\
\hline$E_{\mathrm{s} c}$ & -0.241190 & 0.79695 \\
\hline$E_{\mathrm{p} c}$ & 6.707760 & 6.63291 \\
\hline$E_{\mathrm{S}^{*} a}$ & 19.710590 & 19.80474 \\
\hline$E_{\mathrm{S}^{*} c}$ & 22.663520 & 24.16587 \\
\hline$E_{\mathrm{d} a}$ & 13.031690 & 13.13880 \\
\hline$E_{\mathrm{d} c}$ & 12.748460 & 12.92122 \\
\hline $\operatorname{ss} \sigma$ & -1.645080 & -1.64584 \\
\hline $\mathrm{s}^{*} \mathrm{~s}^{*} \sigma$ & -3.67720 & -2.84245 \\
\hline $\mathrm{s}_{a}^{*} \mathrm{~s}_{c} \sigma$ & -2.207770 & -1.88341 \\
\hline $\mathrm{s}_{a} \mathrm{~s}_{c}^{*} \sigma$ & -1.314910 & -2.78690 \\
\hline $\mathrm{s}_{a} \mathrm{p}_{c} \sigma$ & 2.664930 & 3.02223 \\
\hline $\mathrm{s}_{c} \mathrm{p}_{a} \sigma$ & 2.960320 & 2.95309 \\
\hline $\mathrm{s}_{a}^{*} \mathrm{p}_{c} \sigma$ & 1.976500 & 1.92174 \\
\hline $\mathrm{s}_{c}^{*} \mathrm{p}_{a} \sigma$ & 1.027550 & 1.30469 \\
\hline $\mathrm{s}_{a} \mathrm{~d}_{c} \sigma$ & -2.58357 & -3.03196 \\
\hline $\mathrm{s}_{c} \mathrm{~d}_{a} \sigma$ & -2.320590 & -2.64111 \\
\hline $\mathrm{s}_{a}^{*} \mathrm{~d}_{c} \sigma$ & -0.628200 & -1.84300 \\
\hline $\mathrm{s}_{c}^{*} \mathrm{~d}_{a} \sigma$ & -0.133240 & -1.73510 \\
\hline рр $\sigma$ & 4.150800 & 4.53156 \\
\hline $\mathrm{pp} \pi$ & -1.427440 & -1.86816 \\
\hline $\mathrm{p}_{a} \mathrm{~d}_{c} \sigma$ & -1.874280 & -2.47345 \\
\hline $\mathrm{p}_{c} \mathrm{~d}_{a} \sigma$ & -1.889640 & -1.02836 \\
\hline $\mathrm{p}_{a} \mathrm{~d}_{c} \pi$ & 2.529260 & 2.52741 \\
\hline $\mathrm{p}_{c} \mathrm{~d}_{a} \pi$ & 2.549130 & 2.86419 \\
\hline $\operatorname{dd} \sigma$ & -1.269960 & -1.97058 \\
\hline $\mathrm{dd} \pi$ & 2.505360 & 1.67733 \\
\hline $\operatorname{dd} \delta$ & -0.851740 & -1.58868 \\
\hline$\lambda_{a}$ & 0.172340 & 0.17386 \\
\hline$\lambda_{c}$ & 0.021790 & 0.01589 \\
\hline
\end{tabular}

along with the VCA bands (solid lines). The diamonds plot the mean energies $\bar{E}$ and the error bars indicate the corresponding standard deviations, $\Delta E$, calculated using the following control parameters: minimum gap $(0.05 \mathrm{eV})$, minimum probability $(0.001)$, and minimum band probability (0.50); the standard deviations generally are of the order of $0.01 \mathrm{eV}$. The Lanczos [14] diagonalization of the supercell Hamiltonian searched for eigenstates between -3.0 and $+6.0 \mathrm{eV}$, so there are relatively few projected states at the lower and upper ends of the range; even within the search range, not all eigenstates were returned. Nevertheless the projection algorithm was able to resolve approximate states in the $\Gamma$ - and X-valleys of the lowest conduction band as well as the tops of the upper valence bands fairly well. (For $x=0.85$ the probability to resolve a band at $\mathbf{k}=\mathbf{0}$ fell slightly short of the 0.50 criterion. The value reported in figure 3 below is the band resolved at this slightly lower probability.) The randomalloy and VCA results essentially agree in the valence-band maxima and the conduction-band $\mathrm{X}$-valley. In the $\Gamma$-valley of the lowest conduction band, however, the random-alloy calculation predicts a lower minimum than does the VCA calculation. This tendency was evident in all of the cases we studied: $x=0.15,0.25,0.30,0.40,0.50,0.60,0.70,0.75$, and 0.85 . 
Table 2. Selected energies (in $\mathrm{eV}$ ), $k$-minima (in units of $\pi / a$ ) and effective masses (in units of the free-electron mass) for GaAs and AlAs as reproduced by the parameters of table 1, along with experimental values [17]. Quantities marked with as asterisk $(*)$ are not well-established. Note that [17] reports for AlAs $E_{\text {gap }}^{\Gamma}=2.79-3.03 \mathrm{eV}$; the parameters were optimized to build in an offset $E_{c}^{\mathrm{X}, \min }(\mathrm{AlAs})-E_{c}^{\Gamma}(\mathrm{GaAs})=0.20 \mathrm{eV}$. All masses are computed at the respective extrema.

\begin{tabular}{lllll}
\hline Quantity & GaAs & GaAs-Expt. & \multicolumn{1}{l}{ AlAs } & AlAs-Expt. \\
\hline$E_{c}^{\Gamma}$ & 1.42116 & & 2.47802 & \\
$E_{v}^{\Gamma}$ & -0.00305 & & -0.54281 & \\
$\Delta_{0}$ & 0.32647 & 0.34000 & 0.30915 & 0.30 \\
$E_{c}^{\mathrm{X}, \min }$ & 1.90708 & & 1.62739 & \\
$k_{\mathrm{min}}^{[001]}$ & 1.802 & 1.80000 & 1.667 & 1.80000 \\
$E_{\mathrm{gap}}^{\Gamma}$ & 1.42421 & 1.424 & 3.02083 & 3.02 \\
$E_{\mathrm{gap}}^{\mathrm{X}, \mathrm{min}}$ & 1.91013 & 1.90000 & 2.1702 & 2.153 \\
Electrons $^{\Gamma}$ & & & & \\
$m_{\Gamma}$ & 0.06631 & 0.06700 & 0.13740 & 0.15 \\
$m_{\mathrm{X}, l}$ & 1.69975 & 1.30000 & 0.92276 & 1.1 \\
$m_{\mathrm{X}, t}$ & 0.17512 & 0.23000 & 0.15345 & 0.19 \\
$\mathrm{Holes}_{\mathrm{l}}^{[100]}$ & & & & \\
$m_{\mathrm{lh}}^{[110]}$ & 0.08315 & 0.08710 & 0.16403 & 0.16340 \\
$m_{\mathrm{lh}}^{[111]}$ & 0.07600 & 0.08040 & 0.13417 & 0.13988 \\
$m_{\mathrm{lh}}^{[100]}$ & 0.07414 & 0.07860 & 0.12833 & 0.13471 \\
$m_{\mathrm{hh}}$ & 0.37686 & 0.40300 & 0.42759 & 0.51546 \\
$m_{\mathrm{hh}}^{[110]}$ & 0.65672 & 0.66000 & 1.01798 & 1.09753 \\
$m_{\mathrm{hh}}^{[111]}$ & 0.83905 & 0.81300 & 1.55567 & 1.57033 \\
$m_{\mathrm{so}}$ & 0.16333 & 0.15000 & 0.25785 & $*$ \\
\hline & & & & \\
\hline
\end{tabular}

The lower conduction-band $\Gamma$-valley minimum predicted by the random-alloy calculation has a profound impact on the energy gap as a function of Al mole fraction, as shown in figure 3 . As expected from figure 2, the X-valley gap is essentially the same in the random-alloy and VCA results, both of which agree well with the experimental curve. The $\Gamma$-valley gap is another matter entirely. The VCA results are much larger than the experimental values throughout the range, even at $x=0.15$ and 0.85 . Moreover, the VCA bowing is convex instead of concave, like the experimental curve. As a result the VCA predicts the $\Gamma-\mathrm{X}$ crossover to occur at $x \approx 0.25$, which is much lower than the experimental value, $x \approx 0.43$. The random-alloy result is much closer to the experimental curve and is essentially the same up to $x \approx 0.5$; its $\Gamma-$ $\mathrm{X}$ crossover occurs at $x \approx 0.40$, very near to the experimental value. We attribute much of the difference between the random-alloy and experimental results to the larger AlAs $\Gamma$-valley gap reproduced by our parameters (about $3.03 \mathrm{eV}$ ) versus experiment (about $2.95 \mathrm{eV}$ ). Significantly, the random-alloy curve has the proper convex shape, like the experimental curve. Clearly, the random-alloy calculation predicts gaps in far better agreement with experiment than does the VCA.

In the context of tight binding one can qualitatively understand why the random-alloy and VCA results differ significantly for the $\Gamma$-valley minimum but not the $\mathrm{X}$-valley minimum and the valence-band maximum. The $\Gamma$-valley minimum states are purely s-like (s- and $\left.\mathrm{s}^{*}\right)$. The onsite parameters which differ most between GaAs and AlAs are $E_{\mathrm{s} c}$ and $E_{\mathrm{s}^{*} c}$. Similarly, the nearest-neighbour parameter $\mathrm{s}_{a} \mathrm{~s}_{c}^{*} \sigma$ differs significantly between the two materials, and disorder 


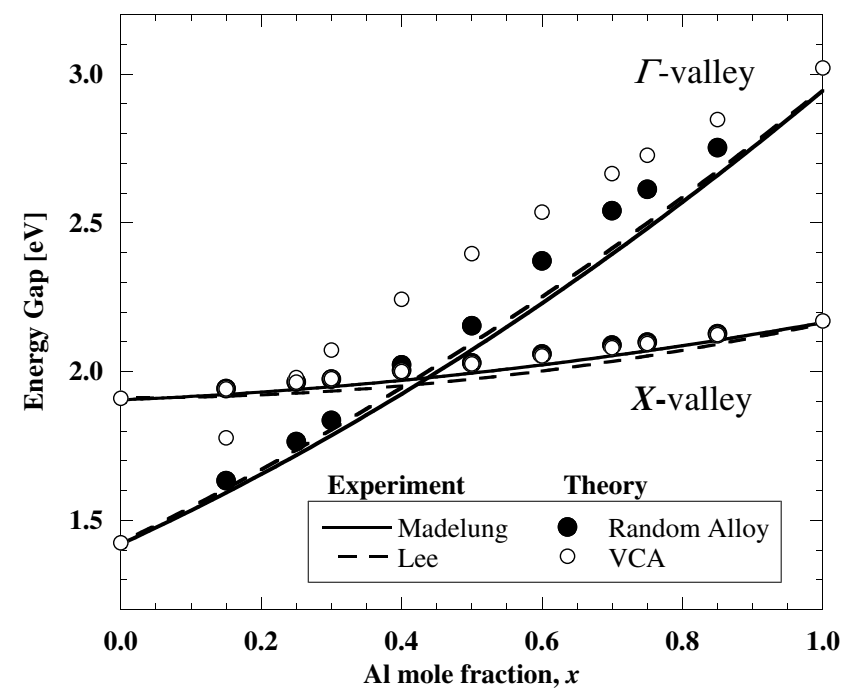

Figure 3. Band gaps versus mole fraction for $\mathrm{Al}_{x} \mathrm{Ga}_{1-x} \mathrm{As}$. Solid circles: gaps based on states projected out of the $40 \times 2 \times 2 \mathrm{Al}_{x} \mathrm{Ga}_{1-x} \mathrm{As}$ random-alloy supercell; open circles: gaps determined from the VCA bands; solid lines: experimental results from Madelung [17]; dashed lines: experimental results from Lee et al [20]. Note the significantly better agreement of the random-alloy results with experiment.

should produce significant deviations from the weighted average. In contrast, the $\mathrm{X}$-valley minimum states are generally a superposition of $\mathrm{s}^{-}, \mathrm{s}^{*}-, \mathrm{p}-$, and $\mathrm{d}$-components, and the valenceband maximum states are purely $\mathrm{p}$ - and d-like. Note that the $\mathrm{p}$ and $\mathrm{d}$ onsite energies are very close in the two materials. There are differences in some nearest-neighbour parameters, but the mixed nature of the $\mathrm{X}$-valley minimum states tends to dilute the effect of any single imbalance. Likewise, in the valence bands the smaller p- and d-parameter differences mean that disorder less strongly affects these states. Because the VCA represents all cations as $\mathrm{Al}_{x} \mathrm{Ga}_{1-x}$ pseudoatoms it should be less successful for the $\Gamma$-valley where disorder accentuates the differences between GaAs and AlAs s-like orbitals versus those of the pseudo-cation.

The focus on $\Gamma$-valley states is motivated by the technological application of AlGaAs as a barrier material in nanodevices such as quantum wells and resonant-tunnelling diodes (RTDs). Evanescent states in AlGaAs connecting the $\Gamma$-valley minimum and light-hole maximum are to a great extent responsible for determining the carrier confinement in GaAs/AlGaAs nanodevices. These states are significant even for GaAs/AlAs RTDs [21].

Because masses and gaps are important in determining proper evanescent state behaviour, we also extract approximate $\mathrm{AlGaAs} \Gamma$-valley masses, shown in figure 4 . The masses for all but $x=0.85$ were found by a best fit of the lowest three $E(\mathbf{k})$ points to the parabola $E=\alpha+\beta k^{2}$; for $x=0.85$ the lowest two were used. A linear fit of the masses is shown and we find

$$
\frac{m^{*}}{m_{0}} \approx 0.069+0.070 x
$$

with a correlation coefficient $R=0.967$. This result agrees well with the experimental fit of Lee et al [20], $m^{*} / m_{0} \approx 0.066+0.088 x$, given our slightly lower AlAs $\Gamma$-valley effective mass. The scatter in the results is due to the uncertainties in the energies, which, as mentioned above, are of the order of $0.01 \mathrm{eV}$. This scatter is simply a consequence of having to calculate masses from $E(\mathbf{k})$ points instead of the more accurate eigenvector method [22], which is available for 


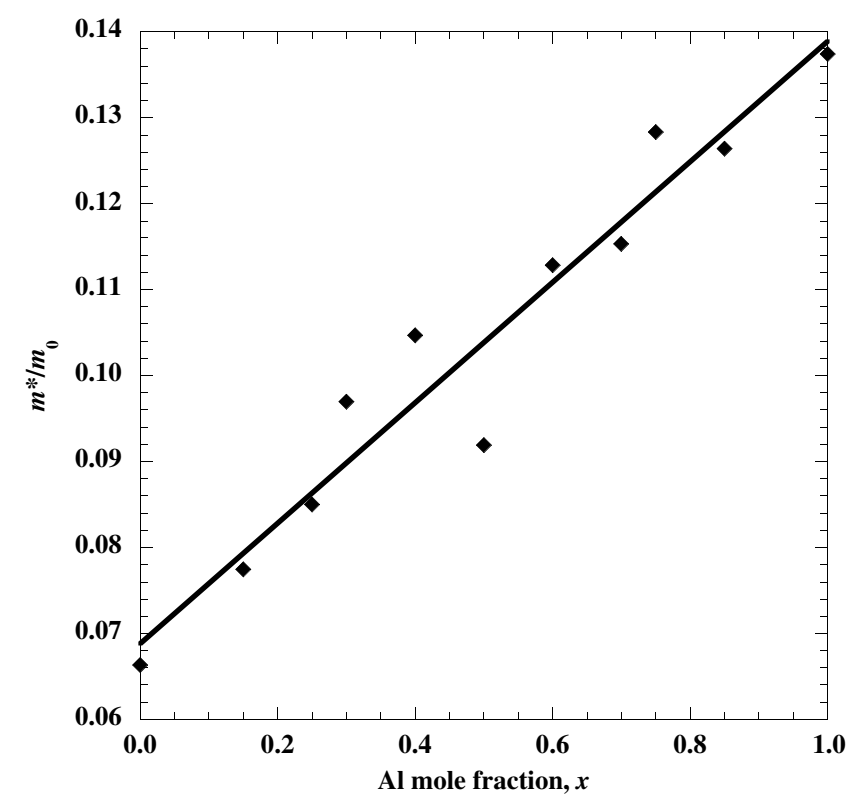

Figure 4. Approximate $\Gamma$-valley effective masses versus mole fraction for $\mathrm{Al}_{x} \mathrm{Ga}_{1-x}$ As. Solid symbols: approximate masses based on states projected out of the $40 \times 2 \times 2 \mathrm{Al}_{x} \mathrm{Ga}_{1-x} \mathrm{As}$ randomalloy supercell; solid line: best linear fit to the data.

only ideal materials. (Significantly reduced scatter would require energy uncertainties about two orders of magnitude less, i.e., smaller than $0.1 \mathrm{meV}$.) The influence of $\Delta E$ on the scatter in the mass implies that some scattering mechanism in the active region of an alloy nanodevice ought to be included when either single-band or VCA tight-binding models are employed to calculate transport.

\section{Conclusions}

The new method presented here significantly extends the capabilities of traditional tightbinding supercell calculations. Previous calculations [5] could identify only band extrema; they could not resolve approximate band dispersions. This method resolves approximate band dispersions permitting the identification of important transport parameters such as satellite valleys and the calculation of approximate effective masses. In the particular case of AlGaAs computed here, the reduction of the bandgap can be clearly identified as contributions of the $\Gamma$-point states. This information allows us to trace the difference back to the GaAs and AlAs orbitals themselves. The results compare very favourably to experimental data at $\mathrm{X}$ and $\Gamma$ while in contrast the VCA shows shortcomings with respect to the bandgap at $\Gamma$. Applications of this method include nanostructures such as quantum wires.

\section{Acknowledgments}

We thank Professor Walter Harrison for discussions and his comments on the manuscript. The work at UAH was supported by Jet Propulsion Laboratory, California Institute of Technology and ONR. The work at Purdue was supported by the National Science Foundation, Grant 
No. EEC-0228390; the Indiana 21st Century Fund; and ONR. Simulations were performed with NCN/nanoHUB.org computational services and hardware ${ }^{4}$.

\section{Appendix}

Here we provide an expanded discussion of the band estimation procedure, relating expressions in the two bases. The estimated bands can be directly related to the expectation values of the full Hamiltonian with respect to the Bloch states. To show this relationship, one starts with the full Hamiltonian for the supercell,

$$
\hat{H}=\sum_{p=1}^{N_{\mathrm{C}} N_{\mathrm{O}, \text { cell }}}\left|\Psi_{p}(\mathbf{K})\right\rangle E_{p}\left\langle\Psi_{p}(\mathbf{K})\right| .
$$

Using equations (3) and (A.1), the energy expectation value of a single small-cell Bloch state at $\mathbf{k}=\mathbf{K}+\mathbf{G}_{\mathbf{n}(j)}$ is

$$
\left\langle\psi_{\eta}\left(\mathbf{K}+\mathbf{G}_{\mathbf{n}(j)}\right)|\hat{H}| \psi_{\eta}\left(\mathbf{K}+\mathbf{G}_{\mathbf{n}(j)}\right)\right\rangle=\sum_{p=1}^{N_{\mathrm{C}} N_{\mathrm{O}, \text { cell }}} E_{p}\left|a_{p ; \eta, \mathbf{n}(j)}\right|^{2} .
$$

Equation (A.2) is of course generally true, whether a band description for the supercell is appropriate or not. If a band description does make sense, then only a few supercell states of similar energies will contribute to the small-cell Bloch state. The sum in equation (A.2) will then run over only a subset $\mathscr{S}(\eta(j))$ of the supercell states:

$$
\left\langle\psi_{\eta}\left(\mathbf{K}+\mathbf{G}_{\mathbf{n}(j)}\right)|\hat{H}| \psi_{\eta}\left(\mathbf{K}+\mathbf{G}_{\mathbf{n}(j)}\right)\right\rangle=\sum_{p \in \mathscr{S}(\eta(j))} E_{p}\left|a_{p ; \eta, \mathbf{n}(j)}\right|^{2} .
$$

If all of these supercell states are available, then the normalization sum

$$
\mathscr{N}_{\eta, j}=\sum_{p \in \mathscr{S}(\eta(j))}\left|a_{p ; \eta, \mathbf{n}(j)}\right|^{2}
$$

should be unity. However, as mentioned above, often only some of the supercell states are available, in which case the normalization is less than unity. Hence, the band energy is calculated as the average

$$
\bar{E}_{\eta(j), \mathbf{n}(j)}=\frac{1}{\mathscr{N}_{\eta, j}} \sum_{p \in \mathscr{S}(\eta(j))} E_{p}\left|a_{p ; \eta, \mathbf{n}(j)}\right|^{2} .
$$

The procedure is readily extended to the case of degenerate or near-degenerate bands. Illustrating it for the case of two near-degenerate bands is sufficient. If the band description is physically reasonable, then only a subset $\mathscr{S}(\eta(j, 1), \eta(j, 2))$ of the supercell states will contribute to the two near-degenerate small-cell Bloch states at $\mathbf{k}=\mathbf{K}+\mathbf{G}_{\mathbf{n}(j)}$. Here the bands to which the Bloch states belong are indexed $\eta(j, l), l=1,2$. In this case, the normalization

$$
\mathscr{N}_{\eta, j}=\sum_{p \in \mathscr{S}(\eta(j, 1), \eta(j, 2))} \sum_{l=1}^{2}\left|a_{p ; \eta(j, l), \mathbf{n}(j)}\right|^{2}
$$

is ideally 2 , but in practice is usually less, since generally not all supercell states are available. The (degenerate) band energy is then just the average of the energy expectation values of the

$4 \mathrm{http} / / /$ nanoHUB.org is a web site dedicated to advancing nanotechnology through theory, modelling, and simulation for research and education. 
two Bloch states,

$$
\begin{aligned}
& \bar{E}_{\eta(j), \mathbf{n}(j)}=\frac{1}{\mathscr{N}_{\eta, j}} \sum_{l=1}^{2}\left\langle\psi_{\eta(j, l)}\left(\mathbf{K}+\mathbf{G}_{\mathbf{n}(j)}\right)|\hat{H}| \psi_{\eta(j, l)}\left(\mathbf{K}+\mathbf{G}_{\mathbf{n}(j)}\right)\right\rangle \\
& \bar{E}_{\eta(j), \mathbf{n}(j)}=\frac{1}{\mathscr{N}_{\eta, j}} \sum_{p \in \mathscr{S}(\eta(j, 1), \eta(j, 2))} \sum_{l=1}^{2} E_{p}\left|a_{p ; \eta(j, l), \mathbf{n}(j)}\right|^{2} .
\end{aligned}
$$

Thus, when the band description of the supercell is appropriate, the average energies represent expectation values of the full supercell Hamiltonian in the Bloch basis.

\section{References}

[1] Klimeck G, Oyafuso F, Boykin T B, Bowen R C and von Allmen P 2002 J. Comput. Mod. Eng. Sci. 3601

[2] Menchero J G and Boykin T B 1999 Phys. Rev. B 598137

[3] Wang J, Rahman A, Ghosh A, Klimeck G and Lundstrom M 2005 Appl. Phys. Lett. 86093113

[4] Rahman A, Klimeck G, Lundstrom M, Boykin T B and Vagidov N 2005 Japan. J. Appl. Phys. 442187

[5] Oyafuso F, Klimeck G, Bowen R C and Boykin T B 2002 J. Comput. Electron. 1317

[6] Canning A, Wang L W, Williamson A and Zunger A 2000 J. Comput. Phys. 16029

Mattila T, Wei S-H and Zunger A 1999 Phys. Rev. B 60 R11245

Mattila T, Wang L-W and Zunger A 1999 Phys. Rev. B 5915270

Kent P R C and Zunger A 2001 Phys. Rev. B 64115208

Wang L-W, Bellaiche L, Wei S-H and Zunger A 1998 Phys. Rev. Lett. 804725

Li J and Wang L-W 2003 Phys. Rev. B 67205319

Kim K, Kent P R C, Zunger A and Geller C B 2002 Phys. Rev. B 66045208

[7] Boykin T B and Klimeck G 2005 Phys. Rev. B 71115215

[8] Rahman A, Guo J, Datta S and Lundstrom M 2003 IEEE Trans. Electron. Devices, IEEE Trans. Nanotechnol. 501853 (joint special issue on nanoelectronics)

[9] Callaway J 1974 Quantum Theory of the Solid State student edn (New York: Academic) section 5.5 Faulkner J S and Stocks G M 1980 Phys. Rev. B 213222

[10] Ujfalussy B, Falukner J S, Moshadam N Y, Stocks G M and Wang Y 2001 Phys. Rev. B 6112005 Wang Y, Stocks G M, Shelton W A and Nicholson D M C 1995 Phys. Rev. Lett. 752867

[11] Biava D A, Ghosh S, Johnson D D, Shelton W A and Smirnov A V 2005 Phys. Rev. B 72113105

[12] Dagram T G, Capaz R B and Koiller B 1997 Phys. Rev. B 569625

[13] Boykin T B, Kharche N and Klimeck G 2006 Eur. J. Phys. 275

[14] Golub G H and van Loan C F 1989 Matrix Computations (Baltimore, MD: Johns Hopkins University Press) chapter 9

[15] Jancu J M, Scholz R, Beltram F and Bassani F 1998 Phys. Rev. B 576493

[16] Slater J C and Koster G F 1954 Phys. Rev. 941498

[17] Madelung O (ed) 1991 Semiconductors: Group IV Elements and III-V Compounds (New York: Springer)

[18] Klimeck G, Bowen R C, Boykin T B, Salazar-Lazaro C, Cwik T A and Stoica A 2000 Superlatt. Microstruct. 2777

Klimeck G, Bowen R C, Boykin T B and Cwik T A 2000 Superlatt. Microstruct. 27519

[19] Adachi S 1985 J. Appl. Phys. 53 R1

[20] Lee H J, Juravel L Y, Wooley J C and SpringThorpe A J 1980 Phys. Rev. B 21659

[21] Boykin T B, Klimeck G, Bowen R C and Lake R 1997 Phys. Rev. B 564102

[22] Boykin T B 1995 Phys. Rev. B 5216317 